\title{
Die Dynamik stadtstrukturellen Wandels in Lateinamerika im Modell der lateinamerikanischen Stadt
}

\author{
Axel Borsdorf, Innsbruck, Wien \\ Jürgen Bähr, Kiel \\ Michael Janoschka, Berlin
}

\section{Einleitung: Vom time-lag bei der Modellierung stadträumlicher Wirklichkeiten}

Seit den ersten Versuchen, die Struktur lateinamerikanischer Städte modellhaft generalisierend wiederzugeben (B̈̈HR 1976; BORSDORF 1976; MERTINS 1980; GORMSEN 1981) und den späteren Modifikationen (B̈̈HR \& Mertins 1981, 1995; Borsdorf 1982, 1994; STEWIG 1983) hat sich das Gefüge der Städte in Lateinamerika wesentlich geändert. Unter dem Einfluss der Globalisierung und ökonomischen Transformation, aber auch aufgrund endogen bedingter sozioökonomischer Veränderungen schwächt sich in vielen Städten die Tendenz zur Polarisierung zwischen den klar voneinander getrennten Vierteln der Reichen (ciudad rica) und den Vierteln der Armen (ciudad pobre) ab. Stattdessen sind völlig neue Muster zu erkennen: Der Trend weist paradoxerweise auf eine sozialräumliche Mischung in großräumiger Betrachtung bei akzentuierter Entmischung (Segregation) auf der Mikroebene. So bilden sich hermetisch abgeschlossene Luxusviertel als Enklaven der Millionäre in durchaus ärmlicher Nachbarschaft, und das diesem Vorgang zugrunde liegende Prinzip räumlicher Fragmentierung lässt sich auch hinsichtlich der Verteilung von Infrastrukturen und Funktionen beobachten. Moderne Einkaufstempel als Kopien nordamerikanischer malls waren noch bis vor wenigen Jahren den Oberschichtvierteln vorbehalten, sie initiierten - wenn sie auf der «grünen Wiese» geplant und implementiert wurden - sogar Planung und Bau von neuen Luxusvierteln (B̈̈HR \& MERTINS 1995: 107). Heute gilt diese räumliche Zuordnung in weit geringerem Maße, da in vielen Großstädten Lateinamerikas derartige malls unabhängig von der räumlichen Konzentration oberer Schichten entstehen.

Es stellt sich daher zu Recht die Frage, ob die bisherigen Modelle der lateinamerikanischen Stadtstruktur und -entwicklung nicht revidiert werden müssen. In diesem Zusammenhang ist zunächst auf einen prinzipiellen Unterschied bei der Konzeption der Modelle hinzuweisen. BäHR, Mertins und beide Autoren gemeinsam zielten auf eine Darstellung der aktuellen Struktur der Stadt, wobei sozialräumliche Muster unter Einbeziehung der Mobilität der Bewohner (Zuwande- rung, intraurbane Migration), z.T. unter qualitativer Beschreibung ihres Sozialstandes, Eingang in die Darstellung fanden. BORSDORF und GoRMSEN dagegen strebten an, den Wandel der Stadtstruktur seit der Kolonialzeit bis zur Gegenwart in Form von Zeitscheiben in ihre Modellbildung einzubeziehen. GORMSENS Modell unterschied sich darüber hinaus von allen anderen, weil die sozialräumliche Struktur nicht im Grundriss, sondern im Profil dargestellt wurde und somit auch physiognomische Aufrisselemente und schematisierte Wertekurven für die Bevölkerungsdichte, den Bodenwert und den Sozialstatus der Bewohner berücksichtigt wurden. Die für die Entstehungszeit seines Modells sehr ausgeprägte Polarisierungstendenz zwischen der ciudad rica und der ciudad pobre findet dadurch eine besonders eindrucksvolle Interpretation. In einem für den Einsatz in der Schule gedachten synoptischen Diagramm (BorsDorf 1994) wurde schließlich versucht, die Zeitscheiben der Modelle von GoRmSEN und BORSDORF zu vereinheitlichen, Grundriss- und Aufrissmodell zu harmonisieren und die wichtigsten ökonomischen, innen- und außenpolitischen sowie gesellschaftlichen Tendenzen als Erklärungsfaktoren für den stadtstrukturellen Wandel in eine Synthese zu bringen. Die Modellentwürfe deutscher Geographen sind auch in der internationalen Literatur diskutiert worden und trugen zur Modifizierung zu stark vereinfachender Strukturschemata bei (DELER 1989; CrowLEY 1995, 1998; FORD 1996).

Zu dieser Zeit entsprach das Modell schon nicht mehr den prinzipiellen Strukturmustern vieler lateinamerikanischer Städte. Deren grundlegender Wandel wurde aber nur zögernd rezipiert. In den monographischen Darstellungen süd- und lateinamerikanischer Städte (Wilhelmy \& BorSDORF 1984/85; GILBERT 1994; BÄHR \& Mertins 1995) finden sich zwar Hinweise auf neue sozialräumliche Segregationstendenzen der Oberschicht, ihre Bedeutung für die Gefügeveränderung der lateinamerikanischen Städte wurde jedoch ebenso wenig erkannt wie in den wenigen Arbeiten, die sich mit den Oberschichtvierteln im Besonderen beschäftigten (ACHILles 1989; KösTER 1995). Erst in jüngeren Untersuchungen brasilianischer Städte (LOPES DE SOUZA 1993; PöHler 1999; Wehrhahn 2000; Coy \& PöHler 2001) fand die Intensivierung sozialräumlicher Segregationsprozesse, verbunden mit der Bildung von gated communities, Beachtung, wobei auf Vorarbeiten brasilianischer Wissenschaftler zurückgegriffen wurde (z.B. Viera Caetano O'Neill 1986; Caldeira 1996). Zeitversetzt erschienen dann auch erste Arbeiten zur Ent- 
wicklung in spanisch-amerikanischen Städten (BorsDORF 1998, 2002a; JANOSCHKA 2000, 2002a; MEYER \& BÄHR 2001). Das Engagement von Diplomanden, die am Forschungsprozess beteiligt waren (PöHLER 1999; Evangelisti 2000; KoHler 2001; Goumas 2002; Janoschka 2002b), muss hierbei hervorgehoben werden.

Es war nur folgerichtig, dass die neu belebte Diskussion um die sozialräumliche Struktur und Entwicklung lateinamerikanischer Städte auch in neue Modelle der Stadtstruktur mündeten. So legten MEYER \& BÄHR 2001 ein Modell der Sozialstruktur Santiago de Chiles vor, BORSDORF präsentierte 2002b ein neues Modell der lateinamerikanischen Stadtentwicklung und JANOSCHKA publizierte ebenfalls 2002a, 2002c den «Vorschlag eines neuen Modells der lateinamerikanischen Großstadt", der von den Ergebnissen seiner Untersuchungen in Buenos Aires ausgeht. Die Notwendigkeit zur Überarbeitung der bisherigen Modellentwürfe begründet er mit neuen, erst in den letzten 30 Jahren entstandenen Strukturelementen, als da sind:

1. die Verbreitung von bewachten Wohnkomplexen für die wohlhabenden Schichten über den gesamten Metropolenraum, die einen klaren Bruch zu der bisherigen sektoralen Anordnung der Oberschichtsviertel darstellen,

2. die Streuung von Einkaufszentren, shopping malls und urban entertainment centers im gesamten Großraum und nicht nur in den traditionellen Sektoren der Oberschicht,

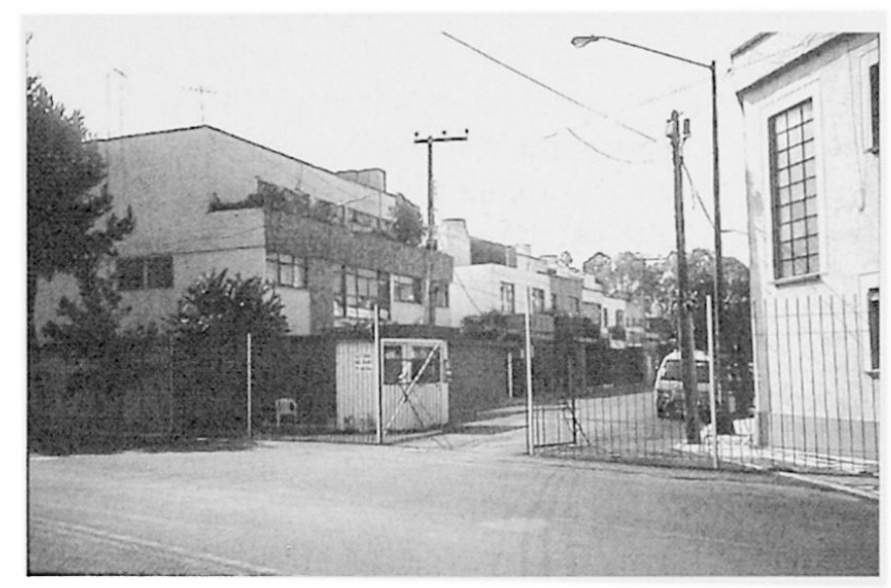

Foto 1: Abgesperrte Strasse in Mexiko-Stadt: Mit Gittern und Schranken wird öffentlicher Raum zum privaten umfunktioniert.

Closed road in Mexico City: Railings and barriers transform public space into private space.

Rue fermée à Mexico-Ville: Avec grilles et barrières, l'espace public est transformé en espace privé.

Foto: A. BORSDORF
3. der Trend zum Bau immer größerer bewachter Wohnkomplexe, die die Größe von Kleinstädten übertreffen können,

4. die gestiegene Bedeutung der Verkehrsinfrastruktur, nach der die Nähe zu einer (Stadt-) Autobahnausfahrt zu einem entscheidenden Aspekt für die Attraktivität des Wohnstandorts wird,

5. die Suburbanisierung der industriellen Produktion durch die Neuansiedlung von Betrieben des sekundären Sektors an der Peripherie, oft in Form von geschlossenen Industrieparks,

6. die zunehmende Abgeschlossenheit und Unbetretbarkeit von Vierteln der Unterschicht und in wachsendem Maße auch der Marginalschicht durch Mauern und Zäune (Foto 1 und 3).

In der Vergangenheit hat sich die Existenz unterschiedlicher Modelle der lateinamerikanischen Stadt als im heuristischen Sinn fruchtbar erwiesen. Da ein Modell bei aller Generalisierung der Wirklichkeit jedoch so nah wie möglich kommen sollte, wird im Folgenden versucht, die bestehenden Ansätze zusammenzuführen und dabei insbesondere die jüngeren Strukturveränderungen zu berücksichtigen.

\section{Reflexionen zur Modellbildung, mit besonderem Bezug zu lateinamerikanischen Städten}

Modelle haben in der Geographie drei Basiskonnotationen. Sie können als Leitbilder anzustrebende Raummuster darstellen, sind demnach konstruktive Utopien. Die Regionalstadt von HiLlebrecht, die Gartenstadt von HowarD oder die Vorstellung einer autogerechten Stadt waren derartige Modelle. Sie können aber auch am Anfang und Ende theoriegeleiteter Arbeit stehen und somit konstitutive Elemente deduktiver Logik bilden. Christallers Zentrale Orte, Boustedts Stadtregion oder die THÜNEnschen Ringe sind Beispiele für diese Auffassung. Modelle können schließlich im phänomenologischen Sinn Reduktionen beobachteter Wirklichkeiten sein, in denen die an vielen Standorten festgestellten Grundstrukturen zu einer einzigen verdichtet werden. Sie bilden häufig den Endpunkt induktiver, aber zuweilen auch hermeneutischer Logik. Die Modelle der lateinamerikanischen Stadt gehören zu diesem Typ. Weil hermeneutische Erfahrungs- und Verständnisprozesse den erkenntnisleitenden Vorgang steuern, ist damit eine gewisse Subjektivität verbunden. Dies erklärt, warum in manchen Modellen und Idealschemata die Strukturen einer einzigen Stadt, auf die dann die Grundmuster der anderen bezogen wurden, noch erkennbar sind.

Der im Folgenden dargestellte Gedankengang versucht zwar, die idiographisch-subjektiven Momente weitgehend in den Hintergrund treten zu lassen, ganz 
kann dies angesichts der Vielgestaltigkeit lateinamerikanischer Städte und der Verschiedenartigkeit der sozioökonomischen und politischen Strukturen und Entwicklungen jedoch nicht gelingen. So muss zu Beginn konstatiert werden, dass insbesondere die Einbeziehung des lusoamerikanischen Kulturraums Schwierigkeiten bereitet. Bei allen Differenzen in der Vergangenheit scheint sich jedoch heute die Stadtstruktur in beiden Kulturräumen stärker anzugleichen, so dass es zumindest für die jüngste Etappe der Stadtentwicklung leichter fällt, das brasilianische Beispiel mit zu berücksichtigen.

Das hier vorgestellte Modell bezieht sich bewusst nicht ausschließlich auf die aktuelle Stadtstruktur, sondern schließt vorangegangene Entwicklungsphasen ein, um die Dynamik der Veränderungen und den Wandel der die Struktur bestimmenden Faktoren sichtbar zu machen. Die vier Hauptphasen der Stadtentwicklung werden durch je ein Schema repräsentiert, das die Stadtstruktur gegen Ende der jeweiligen Epoche zeigt. Grundlage der neuen Modellierung bilden Beobachtungen und die Ergebnisse jüngster Untersuchungen in den Städten Santiago de Chile, Valdivia und Temuco in Chile, Lima und Quito im Andenraum, Mexiko-City und Guadalajara in Mittelamerika sowie Buenos Aires, São Paulo und Rio de Janeiro an der Atlantikküste. Für andere Städte liegen zwar noch keine Untersuchungen vor, dennoch belegen Beobachtungen der Autoren, dass weitgehend parallele Entwicklungen in allen Groß- und Mittelstädten des Kulturerdteils stattfinden.

\section{Die jüngste Phase der lateinamerikanischen Stadt- entwicklung (1970 - heute) im Modell: Die fragmentierte Stadt}

Zwei Raumstrukturationsprinzipien der Vergangenheit sind auch heute noch wirksam: die linear-sektorale Wachstumstendenz und das zellenhafte Wachstum, beide jedoch in einer Weise, die sie von früheren Entwicklungsphasen deutlich unterscheidet. Die Eisenbahn, Leitlinie des linearen Städtewachstums des 19. Jahrhunderts und die wenigen öffentlichen Ausfallstraßen, die, oft als mehrspurige Autobahnen ausgebildet, im 20. Jahrhundert die Rolle der Gleisstränge übernahmen, haben an Bedeutung eingebüßt. Sie konnten mit dem gewachsenen Verkehrsaufkommen nicht Schritt halten (JANOSCHKA 2002a: 65) und wurden in großem Umfang und oft unter Einbeziehung oder gar unter gänzlicher Trägerschaft durch privates Kapital aus- oder neugebaut. Erst die Modernisierung und Ausweitung des intraurbanen Schnellstraßennetzes ermöglichte die Beschleunigung des Verkehrs und machte den sub- und periurbanen Raum in der Fläche auch für die Ober- und Mittelschicht attraktiv
(MeYer \& BäHR 2001: 313). Die Verdichtung des Schnellstraßennetzes bedingte einerseits den Ausbau der gewachsenen linearen Strukturen, war andererseits aber auch die Voraussetzung dafür, dass sich nodale und fragmentierte Strukturen herausbilden konnten, die als das heute bestimmende Strukturationsmuster gelten können.

Auch das zweite, noch wirksame Gliederungsprinzip unterliegt den Rahmenbedingungen der (post)modernen Stadtentwicklung. Zellenhafte Elemente an der Peripherie sind nur noch in manchen Städten (vor allem in den durch Armutsphänomene besonders geprägten wie Lima oder Bogotá) überwiegend als randstädtische Hüttenviertel ausgebildet, in anderen werden sie mehr und mehr von gewaltigen Infrastrukturvorhaben bestimmt, die die bisher bekannten Größenordnungen suburbaner Erschließung sprengen. Aus Buenos Aires sind die megaemprendimientos bekannt, bis zu 1.600 ha große Bauvorhaben mit mehreren tausend Wohneinheiten für die besser verdienenden Bevölkerungsschichten. Das größte dieser Projekte, das Nordelta, soll als ciudadpueblo (Stadtdorf) für bis zu 140.000 Einwohner ausgebaut werden (JANOSCHKA 2002a: 67).

Ähnliche Mega-Projekte gibt es auch in Brasilien. Im Umland von São Paulo ist Alphaville, ca. $30 \mathrm{~km}$ vom Zentrum entfernt, aus dem Boden gestampft worden, ein riesiges ummauertes und abgesichertes Areal, das neben einer eigenen mall und Freizeiteinrichtungen auch Arbeitsplätze in Bürogebäuden für die Bewohner bereitstellt (vgl. Beitrag von CoY \& PöHLER in diesem Heft).

In Chile haben sich - bedingt durch juristische Bestimmungen - auf der grünen Wiese riesige Erschließungsgebiete gebildet, in denen die Fläche jeder Parzelle größer als $5000 \mathrm{~m}^{2}$ ausgelegt wird (MEYER \& BäHR 2001: 303ff.). Im ländlichen Raum, also auch in dem dieser Kategorie zugeordneten Agglomerationsrand, ist eine Grundstücksteilung unter dieser Größenordnung verboten. Diese Bestimmung intendierte die Erhaltung landwirtschaftlicher Mindestgrößen, bildet heute aber für wohlhabende Baulandkäufer kein Hindernis. Rasch erreichen urbanizaciones mit einigen wenigen Siedlern Größenordnungen von 30-50 ha. Es ist jedoch unübersehbar, dass die Käufer derartig großer Grundstücke darauf spekulieren, dass unter dem Sachzwang der Entstehung urbaner Infrastrukturen das Gelände bald als Urbanisierungsfläche deklariert wird, die Parzelle dann geteilt und mit hohem Spekulationsgewinn verkauft werden kann.

Die geschilderten Modifizierungen der linearen bzw. zellenhaften Raummuster sind als Sonderformen jenes Strukturationsprinzips anzusehen, das die heutige 
Dynamik kennzeichnet und die lateinamerikanischen Städte außerordentlich gründlich überprägt. Gemeint ist die Fragmentierung der Stadtorganismen, die sich auf allen Ebenen der Stadtentwicklung durchzusetzen scheint. Fragmentierung ist eine neue Form der Entmischung von Funktionen und sozialräumlichen Elementen, aber nicht, wie früher, im kleinen Maßstab (ciudad rica - ciudad pobre; Wohngebiet - City), sondern im großen, wobei sich kleinere und größere, oft hermetisch abgeschottete funktions- oder sozialräumliche Elemente in einer völlig gegensätzlich strukturierten Umgebung ansiedeln.

Am deutlichsten wird dieser Prozess am Beispiel der Bildung von barrios cerrados. Mit Mauern oder Zäunen umgebene, mit privat finanzierten Sicherheitskräften oder -systemen ausgestattete, zum Teil auch mit hochwertiger, freilich nur den Bewohnern vorbehaltener Infrastruktur (Sport- und Freizeitanlagen, z.T. sogar Bildungseinrichtungen) versehen, bilden diese geschlossenen Viertel hoch segregierte und sozial homogene Inseln im Stadtgefüge (Foto 2). Es handelt sich um Formen freiwilliger sozialräumlicher Segregation (vgl. zur Einordnung in die Segregationstheorie: Borsdorf 2000). Die Grund- und Rahmenbedingungen der Entstehung sind bislang nicht vollständig erforscht. MEYER \& BäHR (2001: 316) und BorsDorf (in diesem Heft, S. 241, Abb. 1) geben erste Erklärungsansätze. Vorläufig kann konstatiert werden, dass als Pullfaktoren das Motiv der Sicherheit (auch für die Kinder), die Möglichkeit des Zusam-

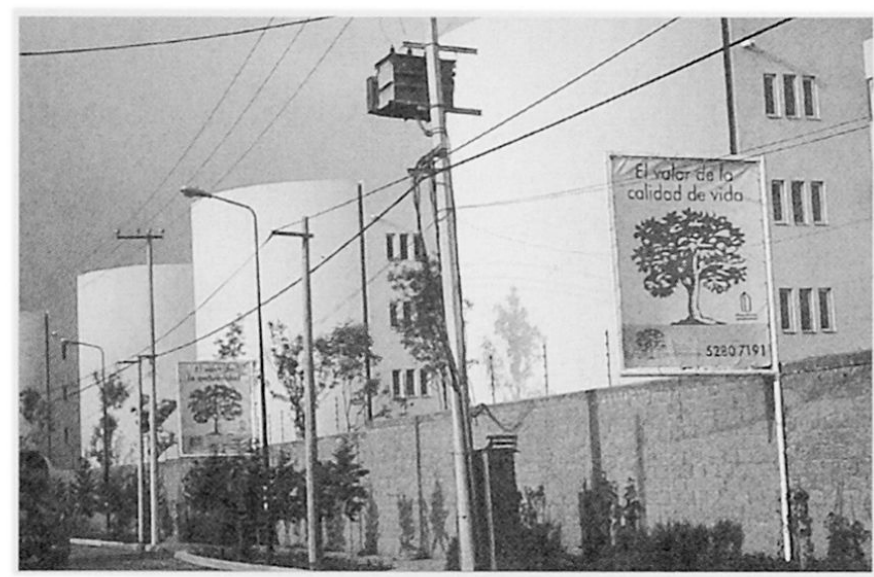

Foto 2: Der Wert der Lebensqualität, der Wert der Exklusivität: Werbung für ein condominio der Luxusklasse

The value of quality of life, the value of exclusiveness: Advertisement for a "condominio" of luxury class

La valeur de la qualité de la vie, la valeur de l'exclusivité: publicité pour un "condominio" de luxe

Foto: A. BORSDORF menlebens mit Menschen der gleichen Sozialschicht, bei den barrios cerrados der Mittel- und Oberschicht auch der Wunsch nach Exklusivität und z.T. der Verwirklichung eines bestimmten Lebensstils eine Rolle spielen, als Pushfaktoren das frühere Wohnumfeld, die Gefahr von Kriminalität oder Terrorismus, die von Medienberichten zusätzlich geschürt und von Immobilienmaklern ausgenutzt wird.

Ein zweites Element der Fragmentierung lateinamerikanischer Städte bilden die Allokationstendenzen funktionaler Raumeinheiten. Besonders klar wird dies am Beispiel des Einzelhandels. In manchen Städten konnte das Stadtzentrum durch Sanierungs-, Verkehrsberuhigungs- und andere upgrading-Maßnahmen (Ladengalerien und -passagen, Metrostationsaus- und -umbau) verlorenes Terrain zurückgewinnen (z.B. Buenos Aires). Im Gefüge der urbanen Einzelhandelsstandorte ist es jedoch selbst im besten Fall nur noch von untergeordneter Bedeutung. Orientierten sich die ersten shopping centers und malls räumlich klar an den neuen suburbanen Villenvierteln der Oberschicht, so ist heute die Standortwahl weit weniger am sozialräumlichen Grundmuster ausgerichtet. Gleichwohl bleiben ärmere Bevölkerungsschichten auch ohne autoritär erscheinende Zwangsmaßnahmen weitgehend ausgeschlossen, weil die shopping centers nur mit dem Auto erreichbar sind (JANOSCHKA 2002a: 68). Zum Teil sind die malls mit Erlebnis- und Aktivitätszentren vom Typ des aus Nordamerika bekannten urban entertainment center verbunden, freilich entstanden solche auch eigenständig. Im Modell werden die malls, entertainment centers und die business parks, die ähnliche Standortpräferenzen haben, aus Gründen der Vereinfachung mit einer Signatur dargestellt. Die gute Erreichbarkeit mit dem privaten PKW ist zu einem entscheidenden Standortfaktor geworden, der bisherige Standortfaktoren scheinbar obsolet macht. Dies gilt nicht nur für die Auswahl des Wohnviertels und den Besuch von Einkaufs- und Freizeiteinrichtungen, sondern auch im Hinblick auf die Allokation von Arbeitsstätten für die Oberschicht. Die neuen Stadtautobahnen wurden daher in das Modell aufgenommen, und es wurde versucht, die Lagekoinzidenzen dieser Schnellstrassen zu barrios cerrados und malls/entertainment centers/business parks darzustellen. Hochwertige Dienstleistungen konzentrieren sich schon seit längerem nicht mehr ausschließlich auf die zentralen Bezirke der Stadt, und auch die Tendenz zur Ansiedlung in den traditionellen Oberschichtvierteln ist bei weitem nicht mehr so dominant wie noch vor ca. 20-30 Jahren.

So prägt die massive Verbreitung von privaten Urbanisierungen, meist in Form der barrios cerrados, im gesamten Stadtgebiet und in der Randzone der Agglomerationen, gepaart mit der ebenfalls dispersen Anord- 
nung von Einkaufs- und Erlebniszentren (malls und urban entertainment centers) sowie von Bürotürmen, die heutige Stadtstruktur. Während alte Industriegebiete vielfach verfallen, bilden sich in manchen Städten an den Flughäfen neue Wirtschaftsschwerpunkte heraus, die an edge cities der USA erinnern. Neben dem exklusiven Beherbergungsgewerbe umfassen sie auch großfächigen Einzelhandel, Erlebnisinfrastrukturen, Logistik und hochwertige, international handelbare Dienstleistungen.

Es hat bereits verschiedene Versuche zur Typisierung der barrios cerrados gegeben. Im Modell wird darauf nicht Bezug genommen, stattdessen werden die vielfältigen Erscheinungsformen zu drei nach Größe, Struktur und Lage unterscheidbaren Typen zusammengefasst. Die sogenannten «urbanen barrios cerrados» sind ummauerte, dicht verbaute, oft in standardisierter (Reihenhaus-) Architektur errichtete Wohnkomplexe, aber auch mit einer Mauer umgebene Appartementhausgruppen oder schließlich nachträglich eingefriedete Straßenzüge. Gemeinsam genutzte Infrastrukturen (Sportanlagen, Schwimmbad etc.) sind kaum vorhanden. Die Bewohner gehören zumeist der Mittel- und oberen Unterschicht an. Suburbane barrios cerrados dagegen sind weit weniger dicht verbaut, mit großzügigen Grünanlagen und Freizeiteinrichtungen versehen. Sie werden in der Regel von Angehörigen der Oberschicht bewohnt. Nur in wenigen lateinamerikanischen Megastädten sind inzwischen auch großflächige barrios cerrados mit integrierter Infrastruktur entstanden, z.B. das Nordelta in Buenos Aires oder Alphaville bei São Paulo. Da aber angenommen wird, dass sich die Tendenz zur Anlage solcher "Stadtdörfer» fortsetzen wird, wurden sie dennoch bereits in das Modell aufgenommen.

Spanisch-amerikanische Städte liegen oft in Becken, lusoamerikanische Großstädte dagegen an der Küste. In beiden Fällen sind die Reserveflächen für Stadterweiterungen großenteils aufgebraucht. Dadurch ist die letzte Stadtentwicklungsphase auch durch die Verdichtung der urbanisierten Fläche gekennzeichnet. Viele der bis 1970 noch inselhaft vor dem urbanen Perimeter angeordneten Sozialbau- und Marginalviertel sind dadurch in das städtische Weichbild integriert worden.

Die Hüttenviertel haben zu einem großen Teil in den letzten Jahrzehnten einen beachtlichen Konsolidierungsprozess durchlaufen. Auch sie wurden vom Trend zur Einfriedung erfasst und sind heute vielfach umzäunt (Foto 3). Wo eine Konsolidierung stattfand, sind die ehemaligen Hütten modernen Gebäuden (oft in Stahlbeton-Skelettbauweise mit gemauerten Wänden) gewichen, die Straßen sind ebenfalls befestigt, Infrastruktur zur Ver- und Entsorgung, Bildungseinrichtungen, Einzelhandel und vielfältige Dienst-

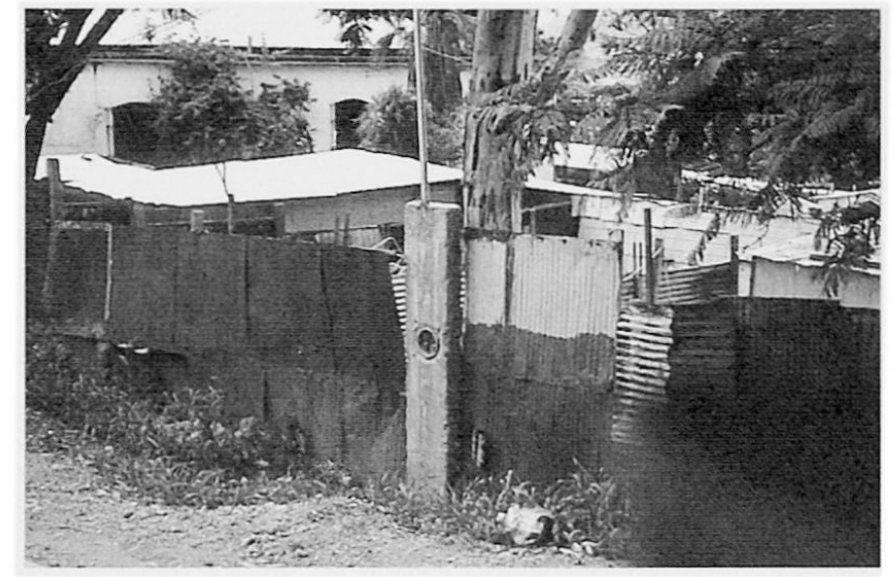

Foto 3: Marginalviertel (barrio perdido) in Mexiko-Stadt: Auch die Hüttenviertel sind auf ihre Art umzäunt. Squatter settlement ("barrio perdido») in Mexico City: The marginal quarters, too, gate themselves in their manner.

Bidonville ("barrio perdido») à Mexico-Ville: les bidonvilles sont enclos à leur façon.

Foto: A. BORSDORF

leistungen sind hinzugekommen, und oft machen die ehemaligen Marginalviertel heute einen durchaus «schmucken» Eindruck. Die in der zweiten Verstädterungsphase noch wichtige Unterscheidung von semilegalen und illegalen Hüttenvierteln (vgl. BÄHR \& MerTINS 1981) ist nicht nur aus Vereinfachungsgründen entfallen, sie ist angesichts der neoliberalen Deregulierung heute nicht mehr bedeutsam. Die in der ersten Hälfte des 20. Jahrhunderts entstandenen, zentral gelegenen Marginalviertel, es handelt sich um die "Gängeviertel» (conventillos, vecindades) und um abgewertete Viertel, werden heute z.T. unter lautstarkem, aber letztlich erfolglosem Widerstand der Bewohner «saniert» oder abgerissen.

Die genannten Entwicklungsprozesse führen zu einer neuen sozialräumlichen und funktionalen Struktur der Städte Lateinamerikas, die als letzte, d.h. vierte Entwicklungsphase im Modell dargestellt ist (Abb. 1). Zum besseren Verständnis sollen im Folgenden aber auch die bisherigen Stadtentwicklungsphasen kurz diskutiert werden. Die Darstellung orientiert sich dabei an der Zeitsequenz und beginnt mit der Gründung und der sich anschließenden kolonialen Epoche.

\section{Parameter der Stadtentstehung und erste räumliche Strukturationsprinzipien im Modell: Die kompakte Stadt}

Die Gründung, Lagewahl und Planung der Städte folgte im spanischen Teil der Neuen Welt spätestens 


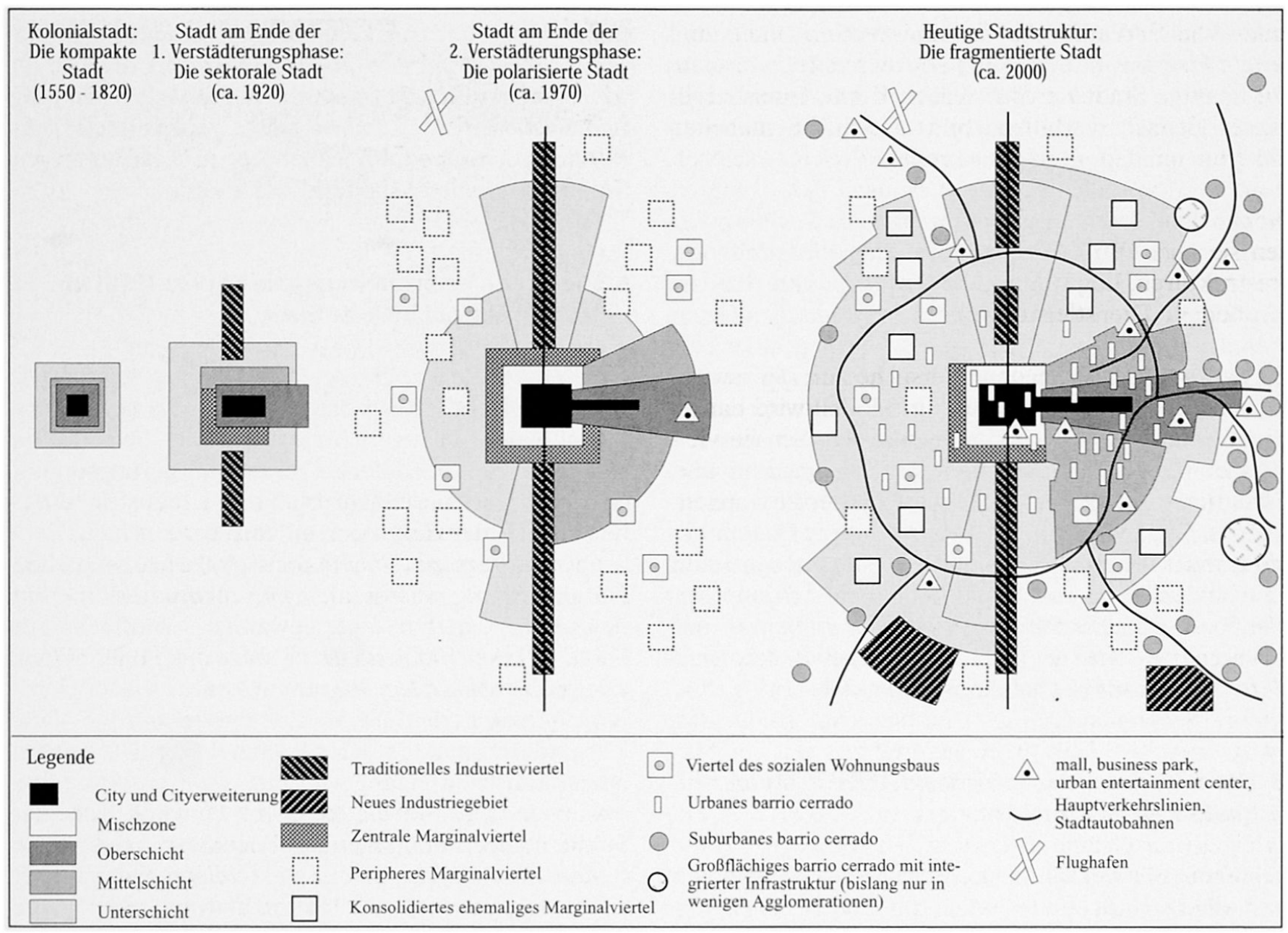

Abb. 1: Modell der Struktur und Entwicklung der lateinamerikanischen Stadt

Model of the structure and development of the Latin American city

Modèle de la structure et du développement de la ville d'Amérique latine

Entwurf: J. BäHR, A. BORSDORF, M. JANOSCHKA

seit 1573 klaren königlichen Anweisungen (WILHELmY \& BORSDORF 1984: 73ff.; BÄHR \& MERTINS 1995: 9ff.). Hauptstädte entstanden im Zentrum der Verwaltungseinheiten in höher gelegenen, klimatisch begünstigten Beckenlagen, lediglich die ihnen untergeordneten Hafenstädte durften an der Küste errichtet werden. Die zentrale, quadratisch angelegte plaza, ursprünglich als Waffenplatz gedacht, bildete den klaren Bezugspunkt, auf den sich das geradlinige, im Schachbrettmuster angelegte Straßennetz orientierte. Die plaza war aber zugleich auch der Mittelpunkt des sozialen Lebens. Nach dem Grad der Entfernung der Wohnung vom zentralen Platz bemaß sich der Sozialstatus des Bürgers. Im sozialräumlichen Muster entsprachen diese Städte dem Modell einer ständisch relativ einfach, dafür aber markant gegliederten Kolonialgesellschaft.

Die Städte hatten im spanischen Kolonialreich vor allem eine Verwaltungsfunktion; der Handel spielte nur eine untergeordnete Rolle. Das übergeordnete ökonomische Ziel, dem auch die Städte zu gehorchen hatten, war der Mehrwerttransfer nach Spanien, d.h. die Ausbeutung von mineralischen und pflanzlichen Rohstoffen und ihr Transport ins ferne Mutterland.

Das dominante Kern-Rand-Gefälle in der sozialräumlichen Struktur spiegelte sich im ringförmigen Aufbau der Stadtviertel. An der plaza wohnten die vornehmsten Familien, oft die Conquistadoren und ihre Nachkommen sowie große Landbesitzer, ferner der Bischof und der von Spanien entsandte Vizekönig, Generalkapitän oder der dem Rang der Stadt entsprechende Spitzenbeamte. Auch der plaza-nahe Straßenkomplex war der Oberschicht vorbehalten. Den folgenden Ring bildeten die Straßenzüge der Mittelschicht, darin lag in der Regel auch die Markthalle. Und 
schließlich wohnten die «armen Weißen», die Indios und Mestizen in den Außenvierteln dieser überaus kompakten Stadt. Zentralisierung, Kern-Rand-Gefälle und schalenförmiger Aufbau können als Strukturationsprinzipien identifiziert werden.

In Brasilien galten in der Frühphase der Stadtentwicklung andere Grundbedingungen. Die dortigen Städte wurden zunächst nur als Küstenstützpunkte für den portugiesischen Warenverkehr in den Indischen Ozean benutzt und mussten daher zum Schutz gegen das Hinterland und konkurrierende europäische Seemächte ummauert werden. Ihr Grundriss war ursprünglich regellos und selbst nach Übernahme der «SchachbrettMode» weniger schematisch als derjenige spanisch-amerikanischer Städte. Anders als diese hatten die lusoamerikanischen Städte von Beginn an wichtige Funktionen im Handel. Auch im Zentrum der brasilianischen Städte entstanden Plätze, und allmählich bildete sich auch dort ein Kern-Rand-Gefälle des Sozialstatus heraus, aufgrund der Küstenlage nicht wie Zwiebelschalen strukturiert, sondern eher halbkreisförmig.

\section{Die erste Verstädterungsphase (1820-1920) im Modell: Die sektorale Stadt}

Die in den ersten Jahrzehnten des 19. Jahrhunderts in fast allen spanischen Kolonien der Neuen Welt errungene politische und ökonomische Unabhängigkeit vom Mutterland bewirkte mit einer gewissen Zeitverzögerung auch eine Umstrukturierung des sozialräumlichen Grundmusters der Städte, während sich im portugiesischen Machtbereich zunächst noch wenig änderte. In viele Staaten Spanisch-Amerikas erfolgte eine in Umfang und nach Herkunft der Migranten verschiedenartige europäische Einwanderung. Von Europa wurden auch die Muster des Städtebaus übernommen, der großzügige Boulevard, in Spanisch-Amerika alameda, prado oder paseo genannt, und die europäische Villa.

In dieser Epoche wurden das Strukturationsprinzip der Linearität und das sektorale Prinzip angelegt, das in der Folge noch ausgebaut und um das Prinzip der Polarisierung erweitert wurde. Da wirtschaftspolitisch zunächst die interne konservative Agraroligarchie bestimmend blieb, gerieten die Schlüsselbereiche der nationalen Ökonomie in die Hände von Ausländern, wobei sich lediglich die Dominanz der Staaten änderte, von Frankreich zu Großbritannien und schließlich immer mehr zu den USA. Die Liberalen, die in fast allen spanisch-amerikanischen Staaten die Konservativen um 1850-70 ablösten, änderten daran wenig, ihr Entwicklungsziel war die Steigerung der Exporte.

Im Modell ist das lineare Gliederungsprinzip in dop- pelter Weise klar zu erkennen: im linearen, später sektoralen Wachstum der Oberschichtviertel, die sich an der Prachtstraße orientieren und in der Entwicklung entlang der Eisenbahn, wo sich Großhandel, Handwerk und erste Produk tionsbetriebe nebst zugehörigen Wohnvierteln der Arbeiterklasse anordnen.

\section{Die zweite Verstädterungsphase (1920-1970) im Modell: Die polarisierte Stadt}

Das Ziel außengerichteter Entwicklung wurde seit den 1920er und 1930er Jahren durch das neue Paradigma einer innengerichteten, auf die Substitution von Importen abzielende Industrialisierungspolitik abgelöst. Durch die Ansiedlung von Industriebetrieben entlang der Ausfallachsen verstärkte sich das lineare Strukturprinzip. Neue Arbeitsplätze in den Städten bei gleichzeitig wachsendem Bevölkerungsdruck auf dem Lande hatten eine gewaltige Landflucht zur Folge (Gans 1992; BäHr \& MerTins 1995: 47ff.). Die große Masse der Migranten konnte weder sozial noch ökonomisch in die Stadt integriert werden. Viele Zuwanderer mussten unter beengten Verhältnissen in innerstädtischen «Gängevierteln» (conventillos, tugurios, vecindades), leben, die den kolonialen Kern der Städte überformten; raumgreifender waren jedoch die randstädtischen Marginalviertel (callampas, barriadas, villas miseria), die als Hüttenquartiere die Städte nahezu einschnürten und nur vor den Sektoren der Reichen Halt machten.

In dieser Zeit entstand der Gegensatz von reicher und armer Stadt, und die Polarisierung wurde zum vorrangigen Strukturationsprinzip. Ergänzend trat jedoch ein weiteres Prinzip hinzu, das des zellularen Wachstums: Auf billig erworbenen Parzellen am Stadtrand errichtete man sog. Viertel des sozialen Wohnbaus, die aber den Wohnraumbedarf ärmerer Schichten bei weitem nicht decken konnten, so dass illegale (Landbesetzungen) und semilegale (nicht genehmigte Parzellierungen) Hüttenviertel zum prägenden Element der Peripherie wurden.

Diese Verstädterungsphase ist in den Modellen der 1970er und 1980er Jahre dargestellt worden. Charakteristisch für diese Epoche war die enorme vertikale und horizontale Mobilität, die in den Modellen vielfach durch Pfeile symbolisiert worden ist. Migrationsströme vom Land in die "Gängeviertel» des Zentrums, von dort in die randstädtischen Marginalviertel, von dort im Zuge vertikaler Mobilität in die Viertel des sozialen Wohnungsbaus kennzeichnen die innerstädtische Mobilitätsdynamik in idealtypischer Weise, wobei auch gegenläufige Bewegungsmuster als Folge von Verelendungs- und Verdrängungsprozessen beobachtet werden konnten (B̈̈HR 1986). 
In den 1970er Jahren wurden auch die ersten shopping centers errichtet, unmittelbar darauf entstanden malls nach nordamerikanischem Muster, die in der Folge zu Kristallisationspunkten für die Neuplanung von Wohnvierteln für die gehobenen Schichten wurden. Während diese Entwicklungen in den älteren Modellen bereits berücksichtigt sind, finden sich noch keine Hinweise auf die beginnende Fragmentierung des Stadtorganismus, obwohl es schon damals abgesperrte Straßen, bewachte Appartement-Hochhäuser und stark segregierte, wenn auch noch nicht ummauerte Lifestyle-Viertel des Country Club-Typs gab.

\section{Schlussbetrachtung: Machen Stadtmodelle noch Sinn?}

Die Erarbeitung von kulturspezifischen Stadtstruktur- und -entwicklungsmodellen ist ein Spezifikum der deutschsprachigen geographischen Forschung. Es ist allerdings zu fragen, ob die Erarbeitung von Modellen, ein typisches Erkenntnisziel der Moderne, der Postmoderne noch angemessen ist. Strukturen lösen sich auf, werden beliebig, die Bedeutung sozioökonomischer Klassen nimmt ab, die Relevanz ganz anders definierter Style-Gruppen nimmt dagegen zu, wobei Zuordnungen schon deshalb vorläufig bleiben, weil Lebensstile sehr schnell gewechselt werden können. Stadt und Land werden dabei unwichtig, die «Zwischenstadt» (SiEverTs 1999) gewinnt an Wertigkeit (vgl. dazu DuboIs-TAINE 2002). Dies sind natürlich Tendenzen in den Industriestaaten, dennoch scheinen sie im Zeitalter der Globalisierung und Internet-Kommunikation rasch von internationalisierten Oberschichtangehörigen als Trendsetter aufgegriffen und auch in Lateinamerika umgesetzt zu werden.

Machen Stadtmodelle also noch Sinn? Bewegen sich nicht im internationalen Maßstab die Erscheinungsformen und -strukturen von Siedlungen so aufeinander zu, dass Städte unterschiedlicher Kulturräume so wenig unterscheidbar werden wie die Ess- und Kleidungsgewohnheiten der Fast-Food- und FranchiseKunden, die in ihnen wohnen? Die Frage nach der Konvergenz oder Divergenz städtischer Strukturen und Prozesse ist schon alt (vgl. Hofmeister 1996; BÄHR \& MERTINS 1995: 1ff.), älter als die gegenwärtige Globalisierungsdebatte. Die Antwort darauf, dass es sowohl variante (kulturraumspezifische) wie invariante (kulturraumübergreifende) Faktoren und Strukturelemente gibt (vgl. LichtenBERGER 1996) hat aber bis heute Bestand. Insofern versteht sich der vorliegende Aufsatz auch als Antithese einer zu stark verallgemeinernden Globalisierungsthese. Zwar hat der Globalisierungsprozess, den wir seit etwa zwei Jahrzehnten nicht nur im Bereich der Wirtschaft, sondern auch in Politik, Kultur, Information und Wissen erle- ben, die weltweite Vernetzung gestärkt und damit in hohem Maße städtische Strukturen beeinflusst, jedoch nicht ausschließlich in Richtung auf eine weltweite Homogenisierung und Vereinheitlichung, sondern auch in Richtung auf lokale Besonderheiten und Spezialitäten, was mit der Begriffsneuschöpfung der «Glokalisierung» umschrieben wird. Das liegt daran, dass in dem System weltweiter Arbeitsteilung die einzelnen Großräume, Länder und Städte eine höchst unterschiedliche Position einnehmen und deshalb die «internationalen Räume», die Teil der globalen Gesellschaft sind und einen bestimmten Lebensstil widerspiegeln, ein quantitativ sehr verschiedenes Gewicht haben. Ebenso schreitet die sozialräumliche Differenzierung unterschiedlich schnell fort und wird in unterschiedlichem Umfang von der beschriebenen «Fragmentierung» abgelöst. Aber auch die Angleichungsprozesse fußen auf unterschiedlichen Traditionen, deren Relikte durchaus noch spürbar sind, nicht nur in den Raumstrukturen, sondern auch in der Lebenswelt ihrer Bewohner. Modelle können die Entwicklungen transparent machen und die Beschäftigung damit kann für das kulturelle Erbe sensibilisieren. So steht am Ende dieses Beitrags doch ein klares Bekenntnis zur Modellbildung und zur fortgesetzten Beschäftigung mit Städten in Lateinamerika.

\section{Dank}

Die Studie wurde finanziert durch den Fonds zur Förderung wissenschaftlicher Forschung, Wien, die Deutsche Forschungsgemeinschaft, den Deutschen Akademischen Austauschdienst und die Humboldt-Ritter-PenckStiftung der Gesellschaft für Erdkunde zu Berlin.

\section{Literatur}

ACHILlEs, G.W.(1989): Strukturwandel und Bewertung sozial hochrangiger Wohnviertel in Rio de Janeiro. - = Tübinger Geographische Studien 104, Tübingen.

BäнR, J. (1976): Neuere Entwicklungstendenzen lateinamerikanischer Großstädte. - In: Geographische Rundschau 28, 4: 125-133.

BÄHR, J. (1986): Innerstädtische Wanderungsbewegungen unterer Sozialschichten und peripheres Wachstum lateinamerikanischer Metropolen. - = Eichstätter Beiträge 18: 143-177.

BäHR, J. \& G. Mertins (1981): Idealschema der sozialräumlichen Differenzierung lateinamerikanischer Großstädte. - In: Geographische Zeitschrift 69, 1:1-33. BäHR, J. \& G. MERTINS (1995): Die lateinamerikanische Großstadt. Verstädterungsprozesse und Stadtstrukturen. $-=$ Erträge der Forschung 288, Darmstadt: Wissenschaftliche Buchgesellschaft.

Borsdorf, A. (1976): Valdivia und Osorno. Strukturelle Disparitäten in chilenischen Mittelstädten. - = Tübinger Geographische Studien 69, Tübingen. 
BorsDorf, A. (1982): Die lateinamerikanische Grossstadt. Zwischenbericht zur Diskussion um ein Modell. - Geographische Rundschau 34,11: 498-501.

BORSDORF, A. (1994): Die Stadt in Lateinamerika. Kulturelle Identität und urbane Probleme. - In: Geographie und Schule 16, 89:3-12.

Borsdorf, A. (1998): Vom Casco Colonial zum Barrio Amurallado: Wohnformen in lateinamerikanischen Städten. - In: Atención! Jahrbuch des Österreichischen Lateinamerika-Instituts 1: 81-105.

Borsdorf, A. (2000): Condominios in Santiago de Chile als Beispiele sozialräumlicher Segregationstendenzen von Ober- und Mittelschicht in lateinamerikanischen Städten. - In: Peripherie 20, 80:25-40.

Borsdorf, A. (2002a): Barrios cerrados en Santiago de Chile, Quito y Lima: tendencias de la segregación socio-espacial en capitales andinas. - In: CABRALES BARAJAS, L.F. (Hrsg.): Latinoamérica: países abiertos, ciudades cerradas. - Guadalajara, Paris: Universidad de Guadalajara, UNESCO: 581-610.

BorsDorf, A. (2002b): La segregación socio-espacial en ciudades latinoamericanas: el fenomeno, los motivos y las consecuencias para un modelo del desarrollo urbano en América Latina. - In: Luzon, J.F. (Hrsg.) (2002b): Documentos del III. Seminario Medamérica. - Barcelona: Universitat de Barcelona, Departament de Geografia Física i Anàlisi Geogràfica Regional (im Druck).

CALDEIRA, T.P.R. (1996): Building up the wall: the new pattern of spatial segregation in São Paulo. - In: International Sociological Science Journal 48: 55-56.

CoY, M. \& M. PöHLER (2001): Wohnghettos der Privilegierten. Die Fragmentierung der brasilianischen Stadt. - In: Matices 8, 29: 8-12.

CROWLEY,W.K.(1995): Order and disorder - a model of Latin American urban land use. - In: APCG (Association of Pacific Coast Geographers) Yearbook 57: 9-31. Crowley, W.K. (1998): Modelling the Latin American city. - In: The Geographical Review 88, 1: 127-130.

Deler, J.P. (1989): Quartiers populaires et structuration de l'espace urbain. Un modèle latino-américain. Pauvretés et développement dans les pays tropicaux. Hommage à Guy Lasserre. - Université de Bordeaux: 475-486.

Dubois-Taine, G. (ed.) (2002): Outskirts of European Cities. - State of the Art Report, COST Action C 10, Brüssel: European Commission.

Evangelisti, B. (2000): Räumliche Segregation, Gated Communities/Condominios in Santiago de Chile. Unveröffentlichte Diplomarbeit, Institut für Geographie, Universität Wien.

ForD, L.R. (1996): A new and improved model of Latin American city structure. - In: The Geographical Review 86, 3: 437-440.

GaNs, P. (1992): Phasen der wirtschaftlichen Entwicklung und ihre Auswirkungen auf das Großstadtwachstum in Lateinamerika. - In: REINHARD, W. \& P. WALD-
ManN (Hrsg.): Nord und Süd in Amerika. - Bd. 1, Freiburg: 212-224.

GILBERT,A. (1994):The Latin American City. - London: Latin American Bureau.

Gormsen, E. (1981): Die Städte in Spanisch-Amerika. Ein zeit-räumliches Entwicklungsmodell der letzten hundert Jahre. - In: Erdkunde 35, 4: 290-303.

Goumas, A. (2002): Sozialräumliche Segregation in lateinamerikanischen Städten am Beispiel der barrios cerrados in Lima, Peru. - Unveröffentlichte Diplomarbeit, Institut für Geographie, Universität Innsbruck.

Hofmeister, B. (1996): Die Stadtstruktur. Ihre Ausprägung in den verschiedenen Kulturräumen der Erde. = Erträge der Forschung 132, 3. Auflage, Darmstadt: Wissenschaftliche Buchgesellschaft.

JANoschKa, M. (2000): Reich und arm in Buenos Aires. Barrios privados als neue Form der Suburbanisierung. - In: Praxis Geographie 30, 12: 60-62.

JANoschKA, M. (2002a): «Stadt der Inseln». Buenos Aires: Abschottung und Fragmentierung als Kennzeichen eines neuen Stadtmodells. - In: RaumPlanung 101: 65-70.

JANOSCHKA, M. (2002b): Wohlstand hinter Mauern. Private Urbanisierungen in Buenos Aires. - = ISR-Forschungsbericht 28, Wien: Verlag der Österreichischen Akademie der Wissenschaften (im Druck).

JANOSCHKA, M. (2002c): Urbanizaciones privadas en Buenos Aires: ¿hacia un nuevo modelo de ciudad latinoamericana? - In: CABRALES Barajas, L.F. (Hrsg.): Latinoamérica: países abiertos, ciudades cerradas. - Guadalajara, Paris: Universidad de Guadalajara, UNESCO: 287-318.

KOHLER,P.(2001): Sozialräumliche Segregation in lateinamerikanischen Städten am Beispiel der barrios cerrados in Quito, Ecuador. - Unveröffentlichte Diplomarbeit, Institut für Geographie, Universität Innsbruck.

KöSTER, G. (1995): Bevölkerungsstruktur, Migrationsverhalten und Integration der Bewohner von Mittelund Oberschichtsvierteln in der lateinamerikanischen Stadt. Das Beispiel La Paz (Bolivien). - = Aachener Geographische Arbeiten 30, Aachen.

LichtenBerger, E. (1996): Stadtmodelle. Reflexionen zur Forschungsgeschichte. - In: Berliner Geographische Studien 44, Berlin: 1-12.

LOPES DE Souza, M. (1993): Armut, sozialräumliche Segregation und sozialer Konflikt in der Metropolitanregion von Rio de Janeiro. - = Tübinger Geographische Studien 111, Tübingen.

Mertins, G. (1980): Typen inner- und randstädtischer Elendsviertel in Großstädten des andinen Südamerikas. - In: Lateinamerika Studien 7: 269-295.

MEYER, K. \& J. BäHR (2001): Condominios in Greater Santiago de Chile and their impact on the urban structure. - In: Die Erde 132, 3: 293-321.

PöHLER, M. (1999): Zwischen Luxus-Ghettos und Favelas. Stadterweiterungsprozesse und sozialräumliche Segregation in Rio de Janeiro: Das Fallbeispiel Barra 
da Tijuca. $-=$ Kleinere Arbeiten aus dem Geographischen Institut der Universität Tübingen 21, Tübingen. SIEVERTS, T. (1999): Zwischenstadt - zwischen Ort und Welt, Raum und Zeit, Stadt und Land. - = Bauwelt Fundamente 118, 3. Auflage, Braunschweig, Wiesbaden.

Stewig, R. (1983): Die Stadt in Industrie- und Entwicklungsländern. - Paderborn: UTB.

Viera Caetano O’Neill, M.M. (1986): Condominios exclusivos: Um estudio de caso. - In: Revista Brasileira de Geografia 48, 1: 63-81.

WeHrhahn, R. (2000): Megastadt São Paulo - Lebensverhältnisse und Umweltbedingungen. - HGO-Journal 15: 105-118.

Wilhelmy, H. \& A. Borsdorf (1984, 1985): Die Städte Südamerikas. Urbanisierung der Erde 3/1 \& 2/2. Berlin, Stuttgart: Bornträger.

\section{Zusammenfassung: Die Dynamik stadtstrukturellen Wandels in Lateinamerika im Modell der latein- amerikanischen Stadt}

Als 1976 die ersten Versuche publiziert wurden, die lateinamerikanische Stadt im Strukturmodell darzustellen, befand sich der Subkontinent volkswirtschaftlich noch unter dem Einfluss der Entwicklungsstrategie der importsubstituierenden Entwicklung bei starker staatlicher Lenkung der Wirtschafts- und Planungsprozesse, auch im urbanen Raum. Seither haben die Länder Lateinamerikas, von Kuba einmal abgesehen, nicht nur einen Richtungswechsel zum Entwicklungsparadigma des Neoliberalismus vollzogen, sie sind auch unter den verstärkten Einfluss von Globalisierungsprozessen geraten. Der Rückzug des Staates bei Privatisierung und Deregulierung erlaubt weitaus mehr Freiheiten. Dies alles hat auch seinen Niederschlag in der Stadtstruktur gefunden, so dass es an der Zeit ist, die Stadtmodelle als Generalisierungen der Wirklichkeit an die neuen Strukturen im Stadtgefüge anzupassen.

Zur Veranschaulichung der Dynamik urbaner Strukturmuster wird dabei die Stadtentwicklung in vier entscheidenden Epochen modelliert, die als Phasen der Kolonialstadt, der sektoralen Stadt, der polarisierten Stadt und schließlich der fragmentierten Stadt identifiziert werden. Dabei wurde versucht, soweit wie möglich die Muster der früheren Modelle zu übernehmen, um dagegen die jüngste Stadtentwicklungsphase herauszustellen.

\section{Summary: The Dynamics of Urban Structural Change in Latin America Reflected in the Latin American Urban Model}

When in 1976 the first models of the Latin American city were published, the subcontinent was still under the economical influence of an industrialisation strat- egy focussing on import substitution. Economic and planning processes, even in urban space, were strongly directed by the State. Since then, all Latin American countries - except Cuba - have adapted an economic paradigm based on neoliberalism. Furthermore, a strong influence of globalisation is apparent. The withdrawal of the State through privatisation and deregulation allow investors, planners and citizens far more liberties. All of these processes have not left the structure of the cities untouched. As models should be generalisations of reality, it was necessary to adapt existing models to reflect current urban structure.

In order to demonstrate the dynamics of urban structural patterns, the urban development model was structured around four fundamental periods, i.e. the colonial town, the sectoral town, the polarised town and, finally, the fragmented town. Thereby, the attempt was made to take over as many elements of former models as possible, in order to emphasise the most recent phase.

\section{Résumé: La dynamique de la mutation structurelle de la ville en Amérique latine, d'après le modèle latino-} américain

Lorsqu'en 1976 furent entreprises les premières tentatives de diffusion du modèle structurel de la ville latino-américaine, ce subcontinent était encore sous l'influence économique d'une stratégie de développement exogène soumise à un fort dirigisme d'Etat tant économique que planificateur, également à l'échelle urbaine. Depuis, les Etats latino-américains, excepté Cuba, n'ont pas uniquement changé de cap en direction du paradigme de développement néo-libéral; ils sont devenus tributaires de l'influence grandissante des processus de mondialisation. Le retrait de l'Etat dans les domaines de la privatisation et de la dérégulation favorise l'émergence de multiples libertés. Cette transformation s'est aussi répercutée sur les structures urbaines, de telle façon que le temps est venu d'adapter le complexe urbain aux modèles urbains en tant que généralisations de la réalité.

La dynamique du développement des modèles structurels urbains est présentée à travers quatre époques déterminantes: celle de la ville coloniale, celle de la ville sectorielle, celle de la ville polarisée et, en fin de compte, celle de la ville fragmentée. L'étude a été menée avec le souci de reprendre le plus d'éléments possibles des modèles antérieurs en vue d'une confrontation avec l'étape actuelle du déploiement urbain.

\section{Didaktische Hinweise}

- Wie lässt sich die Stadtentwicklung Lateinamerikas phasieren, charakterisieren und visualisieren? Welche gesellschafts- und wirtschaftspolitischen Einflüsse führten je zu neuen Phasen? 
- Welches sind die besonderen Elemente der Entwicklung der letzten 30 Jahre und des aktuellen Trends?

- Welches sind die Möglichkeiten und Grenzen von Modellbildungen generell und auf dem Gebiet der Stadtgeographie?

Prof. Dr. Axel Borsdorf, Institut für Geographie der Universität Innsbruck, Innrain 52, A-6020 Innsbruck; Institut für Stadt- und Regionalforschung, Österreichische Akademie der Wissenschaften, Postgasse 7/4/2, A-1010 Wien.

e-mail: Axel.Borsdorf@uibk.ac.at

Prof. Dr. Jürgen Bähr, Geographisches Institut der Christian-Albrechts-Universität, Olshausenstrasse 40, D-24118 Kiel.

e-mail:baehr@geographie.uni-kiel.de

Dipl.-Geogr. Michael Janoschka, Teltower Damm 266, D-14167 Berlin.

e-mail:m.janoschka@berlin.de

Manuskripteingang/received/manuscrit entré le 10.9.2002

Annahme zum Druck/accepted for publication/accepté pour l'impression: 4.12 .2002 